\title{
A survey of Chinese herbal ingredients with liver protection activities Rubin Wang1,2, John Kong ${ }^{1}$, Dali Wang1 ${ }^{1}$, Linda Lin-min Lien ${ }^{1}$ and Eric Jung- chi Lien*1
}

Address: ${ }^{1}$ Department of Pharmaceutical Sciences, School of Pharmacy, University of Southern California, 1985 Zonal Avenue, Los Angeles, CA 90089-9121, USA and 2Amylin Pharmaceuticals, Inc, 9360 Towne Centre Drive, San Diego, CA 92121, USA

Email: Rubin Wang - rbnwang@yahoo.com; John Kong - jokong@calpoly.edu; Dali Wang - delly1984@msn.com; Linda Linmin Lien - elien@usc.edu; Eric Jung-chi Lien* - elien@usc.edu

* Corresponding author

Published: 10 May 2007

Chinese Medicine 2007, 2:5 doi:10.1186/1749-8546-2-5

This article is available from: http://www.cmjournal.org/content/2/I/5

(c) 2007 Wang et al; licensee BioMed Central Ltd.

This is an Open Access article distributed under the terms of the Creative Commons Attribution License (http://creativecommons.org/licenses/by/2.0), which permits unrestricted use, distribution, and reproduction in any medium, provided the original work is properly cited.
Received: 16 November 2006

Accepted: 10 May 2007

\begin{abstract}
A literature survey was conducted on herbs, their preparations and ingredients with reported liver protection activities, in which a total of 274 different species and hundreds of active ingredients have been examined. These ingredients can be roughly classified into two categories according to their activities: (I) the main ingredients, such as silybin, osthole, coumarin, glycyrrhizin, saikosaponin A, schisandrin A, flavonoids; and (2) supporting substances, such as sugars, amino acids, resins, tannins and volatile oil. Among them, some active ingredients have hepatoprotective activities (e.g. anti-inflammatory, anticancer, antioxidant, immunomodulating and liver cirrhosisregulating effects). Calculation of physicochemical parameters indicates that the main ingredients with negative and positive $\mathrm{E}_{\text {lumo }}$ values possibly display their hepatoprotective effects through different mechanisms, such as antioxidative, anti-inflammatory and immunomodulating effects. As the combination of herbs may achieve some treatment effects synergistically and/or additively, it is common in Chinese medicine to use mixtures of various medicinal herbs with pharmacologically active compounds to have synergistic and/or additive effects, or to reduce harmful effects of some pharmacologically active compounds. In particular, the active compounds with Clog P around 2 are suitable for passive transport across membranes and accessible to the target sites. Thus, $\mathrm{E}_{\text {lumo }}$ and Clog $\mathrm{P}$ values are good indicators among the calculated parameters.
\end{abstract}

Seven different physicochemical parameters ( $M W, C \log P, C M R, \mu, E_{\text {homo }}, E_{\text {lumo }}$ and $H_{f}$ ) and four major biological activities (antioxidant, anti-inflammatory, antiviral/antitumor and immunomodulating) are discussed in this review. It is hoped that the discussion may provide some leads in the development of new hepatoprotective drugs.

\section{Background}

It is well recognized that liver is one of the most important organs in the biotransformation of food, drugs, endogenous and exogenous substances. Profuse supply of blood and the presence of many redox systems (e.g. cytochromes and various enzymes) enable liver to convert these substances into different kinds of inactive, active or even toxic metabolites. The burden of metabolism and exposure to dangerous chemicals make liver vulnerable to a variety of disorders, such as acute or chronic inflammation, toxin-/ 
drug-induced hepatitis, cirrhosis and hepatitis after viral infection.

For centuries, many herbs have been used as natural remedies for the prevention and/or treatment of liver diseases. Various herbs and herbal products are believed to have liver protective functions and widely used in clinical practice in the West as well as East. In the First Edition of PDR for Herbal Medicine [1], for example, a total of 49 herbal products were listed under the preparations for clinical use in liver and gall bladder complaints and 32 products for liver disorders. Some complex Chinese herbal formulae, such as Pro-liver Pill (Yanggan Wan)[2], Liver Care (Himalaya Drug Co, Bangalore, India), Liv-52 [3], Jianpi Wenshen Pill (Jianpi Wenshen Wan) [4], Binggan capsules (Binggan Jiaonang) [5], Binggan Tang [6], Yizhu decoction(Yizhu Koufuye) [7], Yiergan Tang [8] and Xiaochaihu Tang (Sho-saiko-to or SST, [9], have been reported to have significant therapeutic effects on liver protection or treatment of liver diseases. It is yet to be established as to which of the herbs and their active ingredients in these formulae contribute most to the activities of liver protection and treatment.

Both manual and electronic search of literature and/or biological information in herbal medicine was carried out in order for us to examine which structural and physicochemical factors probably affect hepatoprotection. Based on two reference books, namely Zhongcaoyao Xiandai Yanjiu [10-12] and The Chemical Constituents of Oriental Herbal Drugs (Vol 1 \& 2) [62,63], electronic literature search was performed with "ovid"(an online resource with access to multiple databases such as Medline, Biosis, Embase, Current Contents, as well as journals and books), by the key words of "herbal medicine with hepatoprotective activity" and was mainly focused on the literature from 1995 to 2005. In this review, most of the reported ingredients of the heptoprotective herbs are summarized and listed under family, genus and species names of the herbs (Additional file 1) and seven physicochemical parameters of the hepatoprotective compounds, namely molecular weight $(\mathrm{MW})$, calculated octanol/water partition coefficient $($ Clog P), molar refraction (CMR), dipole moment $(\mu)$, energy of the highest occupied molecular orbital ( $\left.E_{\text {homo }}\right)$, energy of the lowest unoccupied molecular orbital $\left(\mathrm{E}_{\text {lumo }}\right)$ and heat of formation $\left(\mathrm{H}_{\mathrm{f}}\right)$, have been calculated and reported (Additional file 3 ). Chemical structures of some of the representative compounds together with their respective calculated physicochemical parameters are also provided (Additional file 2).

\section{Biological activities of extracts and ingredients of medicinal herbs}

According to the results from our literature survey, nearly 300 species of plants belonging to 92 different families have been reported to have liver protection or therapeutic effects on hepatic disorders (Additional file 1). Families of Compositae, Labiatae and Leguminosae cover the largest numbers of species.

These plant species usually contain phytochemicals such as phenols, organic acids, flavonoid glycosides, tannins, resins, amino acids, sugars, alkaloids, saponins and volatile oil as well as their own unique constituents. It is not clear whether one, several or all of these components are active ingredients for liver protection. It is likely that some specific ingredients of each herb play a vital role in liver protection/treatment, thereby contributing to their therapeutic effects. Structures of some representative liver protective compounds [10-34] and key physicochemical parameters which may affect their pharmacokinetic and pharmacodynamic effects are provided (Additional file 2).

Among the many hepatoprotective herbs/compounds, milk thistle (Silybum marianum) used in the West [35], glycyrrhizin, Bupleurum chinense (Saiko), Schisandra chinensis (Wuweizi) and Phyllanthus amarus used in traditional Chinese medicine have been most extensively studied and documented [36].

Silymarin, a mixture of flavonolignans extracted from the seeds of Silybum marianum, is most widely used as a remedy for liver diseases. It is composed of four isomers: the major active component silybin, isosilybin, silychristin and silydianin (Figure 1). Moreover, other seed constituents of Silybum marianum such as betaine, other flavonolignans and essential fatty acids from the same plant have been shown to have anti-inflammatory properties [37]. The beneficial effects of silymarin are most often observed in patients with cirrhosis as a result of alcohol abuse [38]. But it does not reduce mortality; nor does it improve liver histology or biochemical markers of liver function among patients with chronic liver diseases [35]. It was reported that treatment with Silybum marianum and its constituents appears to be safe with no signs of adverse effects [15].

Glycyrrhizin (GL), isolated from the water-soluble extract of Glycyrrhiza uralensis, has been orally or intravenously administered for the treatment of chronic viral hepatitis [34]. It was discovered that GL is metabolized into $18 \beta$ glycyrrhetinic acid (GA) and 18ß-glycyrrhetinic acid-3-O$\beta$-D-glucuronide (GAMA) by $\beta$-D-glucuronidase in the liver and intestines $[39,40]$ (Figure 2).

Structure-bioactivity relationship studies indicate that GA is the most active ingredient against platelet aggregation, tumor cell lines, rotavirus infection and growth of Helicobacter pylori, followed by GAMA and GL [41]. Thus, GA is a better hepatoprotective drug than GAMA or GL. It has 
<smiles>COc1cc(C2Oc3ccc([C@@H]4Oc5cc(O)cc(O)c5C(=O)[C@H]4O)cc3OC2CCO)ccc1O</smiles>

Isosilybin<smiles>COc1cc(C2Oc3c(O)cc([C@@H]4Oc5cc(O)cc(O)c5C(=O)[C@H]4O)cc3C2CO)ccc1O</smiles>

Silychristin<smiles>COc1cc(C2Oc3cc([C@@H]4Oc5cc(O)cc(O)c5C(=O)[C@H]4O)ccc3OC2CO)ccc1O</smiles>

Silybin<smiles>COc1cc(C2C3CO[C@@]4(O)C(=O)C5C=C([C@H]6Oc7cc(O)cc(O)c7C(=O)[C@@H]6O)C2C5C34)ccc1O</smiles>

Silydianin

Figure I

Structures of the four isomers in silymarin.

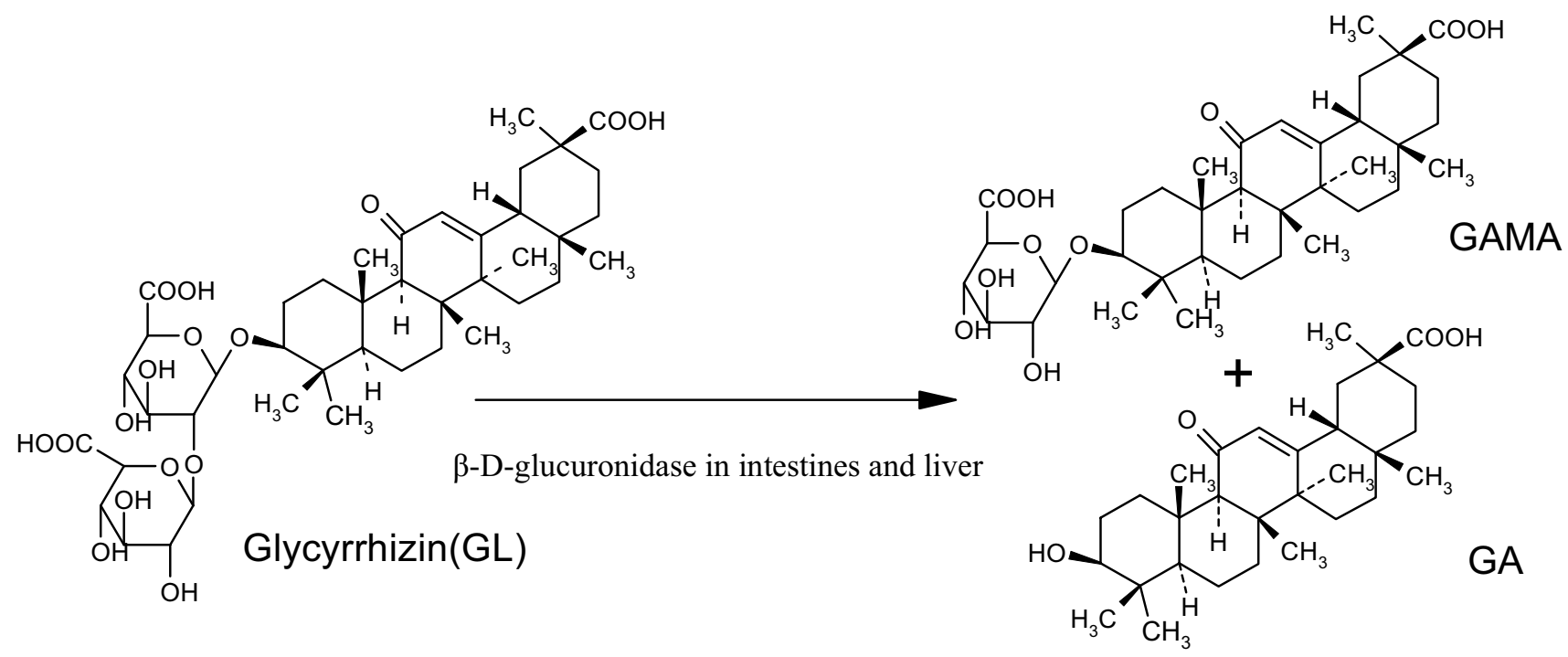

Figure 2

Metabolism of glycyrrhizin catalyzed by $\beta$-D-glucuronidase in human liver and intestines. 
been suggested that GL (a natural prodrug and sweet principal of Glycyrrhiza) and its hydrolytic metabolites (GAMA, GA) bind to hepatocytes to modify the expression of HBV (hepatitis B virus)-related antigens and suppress sialylation of hepatitis $B$ virus surface antigen (HbsAg) [34,42]. Clinical studies showed that GL has comparable antiviral effects to those of interferon and that it is effective in reducing complications of chronic hepatitis C without apparent side-effects $[43,44]$.

Schisandra chinensis (Wuweizi), traditionally used as a tonic, is now widely used for the treatment of chemical/ viral hepatitis. Biological studies indicate that seed extract of Schisandra chinensis can enhance hepatic GSH (glutathione) antioxidant/detoxification system and facilitate both processes in the liver. It may be a promising agent for improving phase I oxidative metabolism in liver damaged by $\mathrm{CCl}_{4}[45]$. Chemical investigations on the extract have revealed the presence of lignans with a dibenzocyclooctadiene skeleton such as schisandrin, gomisin A, deoxyschisandrin (schisandrin A), $\gamma$-schisandrin and wuweizisu C [46] (Figure 3). These components are believed to be active in hepatoprotective, anti-inflammatory, anticancer, anti-HIV and immunomodulating effects [47-50].

Structure-activity relationship (SAR) analyses suggest that the presence of methylenedioxy/a hydroxyl group in the dibenzocyclooctadiene skeleton is an important structural feature for hepato- and neuro-protective activities [51,52]. Osthole, a coumarin derivative contained in many medicinal plants especially in Boenninghausenia albiflora, was reported to protect against liver injury, and these effects are related to the presence of 7-methoxy and 3-methyl-2butenyl groups in the structure $[53,54]$. Due to its low toxicity, osthole appears to be suitable for the treatment of chronic hepatitis and can be used as a lead compound for further development of orally ingested hepatoprotective drugs.

Apart from single herbal medicine, 'Kampo medicine' which utilizes combinations of raw herbs, was widely used in Japan, among which SST is the most well-known. It is a spray-/freeze-dried extract from seven medicinal herbs (Bupleurum falcatum, Glycyrrhiza glabra, Panax ginseng, Pinellia ternate, Scutellaria baicalensis, Zizyphus jujube and Zingiber officinale). Many clinical and experimental studies demonstrated that SST has a variety of therapeutic effects ranging from anti-inflammatory, antioxidant immunomodulating to hepatoprotective, especially on chronic hepatitis. HPLC (high performance liquid chromatography) analysis of pharmacologically active ingredients in SST indicated that the major fractions of SST contain compounds of flavonoid-like structures (e.g. baicalin, baicalein, liquiritin) and triterpene saponins (GL, saikosaponins b1 \& b2, ginsenosides Rg1 and Rb1) [55]
(Figure 4). Comparative experiments showed that the liver concentrations of the active constituents of SST extract in the liver-injured rats increased significantly after the animals received SST extract, as compared with the groups receiving the respective purified constituents. Thus, administration of SST extract was more useful than that of its active ingredients individually [56].

In summary, the ingredients as stated above possess hepatoprotective activities, ranging from antiviral, antiinflammatory, anticancer, antioxidant to immunomodulating and liver cirrhosis regulating effects. Why do the active constituents with different structural features have common biological activities? There may be some innate factors affecting liver protective activities.

\section{SAR analysis of some hepatoprotective ingredients}

A total of 82 representative compounds belonging to nine major groups (i.e. oleanolic acids, hederagenins, polysaccharides, rehmanniosides, xanthine derivatives, alkenes \& alkanes, camelliagenin \& theasapogenol, campenosides \& flavonoids, and aromatic compounds) have been reported to have hepatoprotective effects (Additional file 3). Seven different physicochemical parameters, namely the Clog P, CMR, $\mu, \mathrm{E}_{\text {homo }^{\prime}} \mathrm{E}_{\text {lumo' }}, \mathrm{H}_{\mathrm{f}}$ and MW, have been selected as molecular indicators:

(1) $\mathrm{E}_{\text {lumo }}$ values can be divided into three subsets ranging from $-1.12 \sim-0.10$, and $0.03 \sim 1.07$, to $1.10 \sim 2.12$ (Additional files 2 and 3 ). As $\mathrm{E}_{\text {lumo }}$ values reflect electron affinity of the respective compounds [57], these positive and negative ranges may indicate different mechanisms of actions in hepatoprotection. For example, the $\mathrm{E}_{\mathrm{lumo}}$ values of Silybum marianum, osthole, coumarin, as well as flavonoids and their glucosides, ranging from -1.03 to -0.83 , suggest that their hepatoprotective activities may be achieved via their antioxidative properties, while glycyrrhizin, Schisandra chinensis and Bupleurum chinense, with their $\mathrm{E}_{\text {lumo }}$ values ranging from 0.03 to 1.07 , possibly exert their liver protection through other mechanisms (e.g. anti-inflammatory and/or immunomodulating).

(2) Clog P value, a measure of the hydrophobicity of the compound, may contribute to hydrophobic interactions when the compound binds to the receptor. Different series of compounds vary considerably in their ClogP values (Additional file 3). Polysaccharides, rehmanniosides, rutin, caffeine and theophylline have negative Clog P values ranging from -0.06 to -7.82 . Conversely correlated with the number of sugar moieties attached to the corresponding structures, the Clog P values of camelliagenins, theasapogenols, oleanolic acids and hederagenins are mostly above 4 . The presence of sugar units decreases the partition coefficient of the core structures in the direction 
<smiles>[R]C1(C)Cc2cc(OC)c(OC)c(OC)c2-c2c(cc(OC)c(OC)c2OC)C[C@H]1C</smiles>

$\mathrm{R}=\mathrm{OH} \quad$ Schisandrin $\mathrm{R}=\mathrm{H}$<smiles>[R]C1(C)Cc2cc(OC)c(OC)c(OC)c2-c2c(cc3c(c2OC)OCO3)C[C@H]1C</smiles>

Gomisin A<smiles>COc1c2cc(c3c1OCO3)-c1c(cc3c(c1OC)OCO3)C[C@H](C)[C@H](C)C2</smiles>

\section{Osthole}<smiles>COc1ccc2ccc(=O)oc2c1CC=C(C)C</smiles>

Wuweizisu $\mathrm{C}$

Figure 3

Structures of the dibenzocyclooctadiene compounds and osthole.

of the values getting closer to the ideal $\log \mathrm{P}_{0}$ of 2 and facilitates transmembrane absorption in human body before the compounds' binding to the receptors [58-60]. Other series of compounds have Clog P between 0.7 and 4.0. The more active compounds in hepatoprotection have more favorable Clog P values around 2, such as silybin (1.94), glycyrrhizin (1.89) and coumarin derivatives (1.4 3.7), which are suited for passive transport across membranes and make it easier for the compounds to access the target sites (Additional file 2).

(3) An indicator of the potential of dipolar interactions among molecules, $\mu$ may have some effects on transmembrane absorption as well as interactions with receptors once the molecules are administered and transported via body fluid. The dipole moment values can be roughly divided into three subgroups: high $(9.26-6.12)$, medium $(5.98-1.62)$ and low $(1.45-0.24)$ (Additional file 3$)$. It was anticipated that compound with high or low value of dipole moment would not possess ideal biological activity because it was difficult to cross multiple biological membranes to reach active sites. Some prodrugs with high values of dipole moment (glycyrrhizin: 7.01) may still display some biological activities after metabolic removal of the polar group (18 $\beta$-Glycyrrhetinic acid: 5.83 ). Only the compounds with medium dipole moment values possibly have satisfactory hepatoprotective activities (silybin: 1.92; osthole: 3.77). Finally, $\mathrm{MW}$ and $\mathrm{H}_{\mathrm{f}}$ values of these compounds may have some effects on their biological activities in their own ways (Additional file 3).

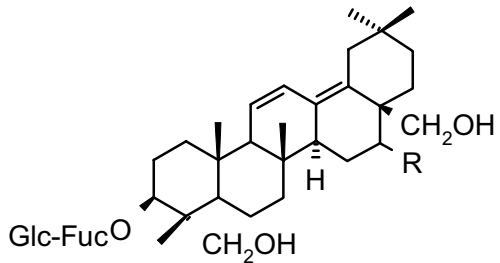

$$
\begin{aligned}
& \text { Saikosaponin } b_{1} R=---O H \\
& \text { Saikosaponin } b_{2} R=-O H
\end{aligned}
$$

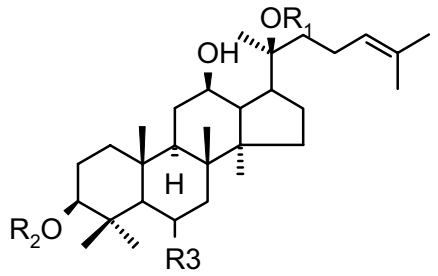

Ginsenoside $\mathrm{Rb}_{1}: \mathrm{R}_{1}=\mathrm{R}_{2}=$ glc-; $\mathrm{R}_{3}=\mathrm{H}$

Ginsenoside $\mathrm{Rg}_{1}: \mathrm{R}_{1}=$ glc-; $\mathrm{R}_{2}=\mathrm{H} ; \mathrm{R}_{3}=---\mathrm{O}-\mathrm{glc}$

\section{Figure 4}

Structures of the saikosaponin and ginsenoside compounds. 


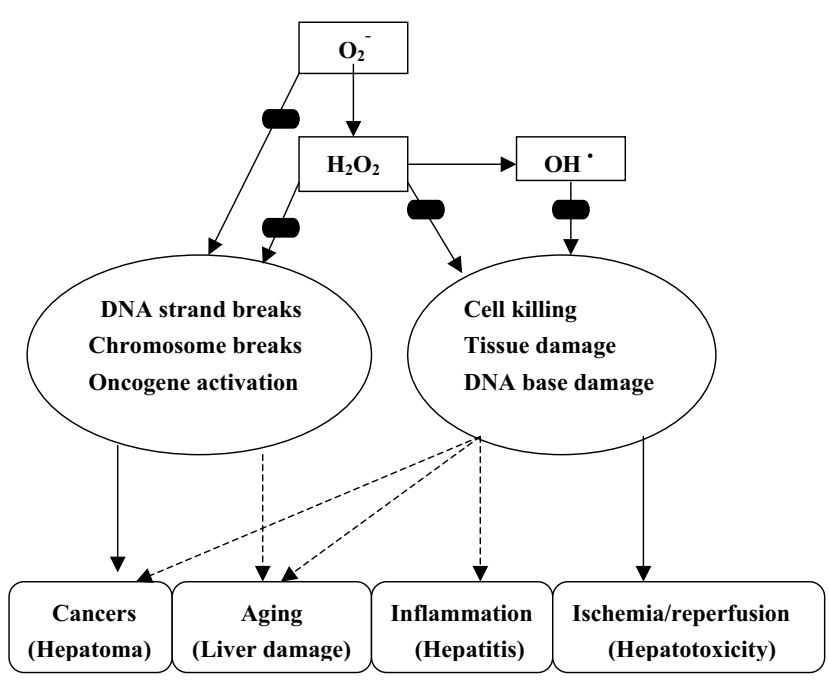

Figure 5

Damaging effects of oxyradical on biological systems and sites of blockades ( ) by antioxidants/free radical scavengers (adapted and expanded from reference [6I]).

Although biological effects of these ingredients have not been fully elucidated by these data, the antioxidant activities are indicated in their $\mathrm{E}_{\text {lumo }}$ values. It is speculated that the compounds with negative $\mathrm{E}_{\text {lumo }}$ values may act as free radical scavengers by having one electron from the highest occupied molecule orbital (HOMO) changed to the lowest unoccupied molecule orbital (LUMO), thereby eliminating the damaging effects of free radicals. The damaging effects of oxidation on the biological system via radical mechanism and the hepatoprotective effects by antioxidants and/or free radical scavengers are illustrated in Figure 5[61].

\section{Conclusion}

Herbal medicine has been used in China for more than two thousand years and is now being gradually accepted worldwide, particularly in Asia, Europe and the North America. Differences, however, do exist between the East and West in the areas such as philosophical view point, concept of diseases and treatment approaches. In the West, people tend to use a single herb in therapies and try to identify active ingredients and therapeutic mechanisms, whereas in Chinese medicine it is common to use mixtures of various medicinal herbs which contain a range of pharmacologically active compounds working additively/synergistically to treat patients or to reduce harmful effects of some chemical compounds. It is our hope that the integration of Chinese and Western medicine will not only identify different ingredients with similar bioactivities or similar ingredients with different bioactivities, but also provide us with methods for quality control in herbal medicine and clues to elucidate action mechanisms of active ingredients.

This review is a summary of 274 species (in 216 genera among 92 families) of herbs that have been reported to have positive effects on liver protection and treatment, as well as hundreds of active ingredients. These ingredients can be roughly classified into two categories: (1) the main ingredients, such as silybin, osthole, coumarin, glycyrrhizin, saikosaponin A, schisandrin A, flavonoids, and (2) the supporting substances, such as sugars, amino acids, resins, tannins and volatile oil. Calculations of molecular parameters suggest that negative and positive $\mathrm{E}_{\text {lumo }}$ values in the main ingredients represent two different hepatoprotective mechanisms. The main ingredients with negative $\mathrm{E}_{\text {lumo }}$ values may display their hepatoprotective effects through an antioxidative mechanism, whereas other ingredients with positive $\mathrm{E}_{\text {lumo }}$ values may act via an antiinflammatory and/or immunomodulating mechanism. Moreover, the Clog P values of many pharmacologically active compounds are around two and other ingredients with higher or lower Clog P values usually go through metabolic processes in which some hydrophilic groups are added or removed so that their Clog $\mathrm{P}$ values are around two. $\mathrm{E}_{\text {lumo }}$ and Clog P values, therefore, should be useful in the future development of hepatoprotective drugs.

\section{Abbreviations}

MW:Molecular weight

Clog P: Calculated octanol/water partition coefficient

CMR: Molar refraction

$\mu$ : Dipole moment

$\mathrm{E}_{\text {homo }}$ : Energy of the highest occupied molecular orbital

$\mathrm{E}_{\text {lumo: }}$ : Lowest unoccupied molecular orbital

$\mathrm{H}_{\mathrm{f}}$ : Heat of formation

SST: Sho-saiko-to

GL: Glycyrrhizin

GAMA: $18 \beta$-glycyrrhetinic acid-3-O- $\beta$-D-glucuronide

HBV: Hepatitis B virus

HbsAg: Hepatitis B virus surface antigen

GSH: Glutathione 
SAR: Structure-activity relationship

HPLC: High performance liquid chromatography

HOMO: Highest occupied molecule orbital

LUMO: Lowest unoccupied molecule orbital

\section{Competing interests}

The author(s) declare that they have no competing interests.

\section{Authors' contributions}

RW compiled all data and conducted the computation of the physicochemical parameters used and writing of the manuscript. JK and DW assisted in the literature search and the computation of the parameters used. LLL and EJL contributed equally in the editing of the manuscript and literature survey/interpretation.

\section{Additional material}

\section{Additional File 1}

Plants reported to have liver protection activities. The table provides genus and species names of the hepatoprotective herbs, and their reported ingredients.

Click here for file

[http://www.biomedcentral.com/content/supplementary/17498546-2-5-S1.doc]

\section{Additional File 2}

Representative structures of compounds with potential hepatoprotection and their key physicochemical parameters (MW, Clog P,CMR, $\mu$ and $\left.E_{\text {lumo }}\right)$. The table presents the chemical structures of the compounds with potential liver protection and the corresponding physicochemical parameters, namely $\mathrm{MW}, \mathrm{Clog} P, C M R, \mu$ and $E_{\text {lumo. }}$.

Click here for file

[http://www.biomedcentral.com/content/supplementary/17498546-2-5-S2.doc]

\section{Additional File 3}

The hepatoprotective compounds and the calculated physicochemical parameters used in analysis. The data provided represent the physicochemical properties of the compounds with potential hepatoprotection. Click here for file

[http://www.biomedcentral.com/content/supplementary/17498546-2-5-S3.doc]

\section{Acknowledgements}

This work is dedicated to the fond memory of the late Prof and Mrs HongYen Hsu for their introduction of Chinese herbal medicine to EJL and LLL. The authors also express their gratitude to the $\mathrm{H} \& \mathrm{~L}$ Charitable Foundation for its generous support over the years.

\section{References}

I. Gruenwald J, Brendler T, Jaenicke C, Eds: PDR for Herbal Medicines. Montvale, NJ: Medical Economics Co, Inc; 1998.
2. Yang M, Chen K, Shih JC: Yang-Gan-Wan protects mice against experimental liver damage. Am J Chin Med 2000, 28: I55-162.

3. Huseini HF, Alavian SM, Heshmat R, Heydari MR, Abolmaali K: The efficacy of Liv-52 on liver cirrhotic patients: a randomized, double-blind, placebo-controlled first approach. Phytomedicine 2005, 12:619-624.

4. Song YW, Song YZ: Observation of Chinese herbal treatment based on 'spleen' and 'kidney' on asymptomatic carriers of hepatitis B virus. Zhejiang Zhongyi Zazhi I994, I 2(5):487-488.

5. Han GP, Wang ZY, Peng SL: Binggan capsule combined with interferon for treatment of $\mathbf{3 0}$ cases of hepatitis $\mathbf{C}$. Henan Zhongyi 1997, I 2(5):43-44.

6. Pei ZG, Liu YL, Zhao SM: Therapeutic observation on Binggan decoction combined with interferon in 46 cases of hepatitis C. Shiyong Zhongxiyi jiehe Linchuang 1996, 9(7):444.

7. Jiang $Y H$ : Treatment of $\mathbf{2 0}$ cases of hepatitis $\mathbf{C}$ using a self-prescribed Yizhu oral liquid. Nanjing Zhongyiyao Daxue Xuebao 1999. I5(4):256.

8. Yu W: Yi Er Gan decoction for treatment of $\mathbf{4 0}$ cases of hepatitis C. Xin Zhongyi 1995, 27(8):46-47.

9. Yamashiki M, Nishimura A, Suzuki H, Sakaguchi S, Kosaka Y: Effects of the Japanese herbal medicine "Sho-saiko-to (TJ-9)" on in vitro interleukin- 10 production by peripheral blood mononuclear cells of patients with chronic hepatitis C. Hepatology 1997, 25: 1390-1397.

10. Institute of Materia Medica Chinese Academy of Medical Science: Zhongcaoyao Xiandai Yanjiu. 1997, I:350.

II. Institute of Materia Medica Chinese Academy of Medical Science: Zhongcaoyao Xiandai Yanjiu. 1997, 2:160-182.

12. Institute of Materia Medica Chinese Academy of Medical Science: Zhongcaoyao Xiandai Yanjiu. 1997, 3:63-86.

13. Institute of Materia Medica Chinese Academy of Medical Science: Zhongcaoyao Xiandai Yanjiu. 1997, I:188-203.

14. Lien EJ, Li WY: A survey of Chinese herbs V: Artemisia capillaries Thunb. (yinchen, Inchinko). Bull Orient Healing Arts Inst of USA 1983, 8: 18-25.

15. Morazzoni P, Bombardelli E: Silybum marianum (Carduus marianus). Fitoterapia 1995, 46(I):3-42.

16. Li WY, Lien Ej: A survey of Chinese herbs and Phytopharmaceuticals Boca Ralton: CRS press; 1989:121-125.

17. Higuchi R, Kawasaki T: Pericarp saponins of Akebia quinata Decne: Glycosides of hederagenin and oleanolic acid. In Chem Pharm Bull Volume 24. Issue 5 Tokyo; 1976:1021.

18. Aoki $\mathrm{T}$, Tanio $\mathrm{Y}$, Suga $\mathrm{T}$ : Triterpenoid saponins from Fatsia japonica. Phytochemistry 1976, I 5:78I-784.

19. Higuchi R, Kawasaki T: Pericarp saponins of Akebia quinata Decne: Glycosides of hederagenin and oleanolic acid. In Chem Pharm Bull Volume 24. Issue 6 Tokyo; 1976:13|4.

20. Oshio H, Naruse $Y$, Inouye $H$ : Quantitative analysis of iridoid glycosides of Rehmanniae radix. Shoyakugaku Zasshi 198I, 35:291-294.

21. Hasegawa T, Koike K, Takahashi S, Ariyoshi U: Constituents of leaves and roots of Kailei jio (Rehmanniae glutinosa Libosch. Forma hueichingensis; Hsia). Shoyakugaku Zasshi 1982, 6:I.

22. Institute of Materia Medica Chinese Academy of Medical Science: Zhongcaoyao Xiandai Yanjiu 1996, 2:I-5I.

23. Talapatra SK, Mukhopadhyay SK, Talapatra B: Minor coumarins of Boenninghausenia albiflora. Phytochemistry 1975, 14:836-37.

24. Talapatra SK, Mukhopadhyay SK, Talapatra B: Nodakenetin acetate: A new coumarin from Boenninghausenia albiflora. Phytochemistry 1973, | 2:23|2-14.

25. Nayar MNS, Bhan MK, George V: A new coumarin in Boenninghausenia albiflora. Phytochemistry 1973, I 2:2073-74.

26. Miyazaki T, Mihashi S: Studies on the constituents of Boenninghausenia albifloria Meissner Var. Japonica S. Suzuki. I. Structure of matsukaze-lactone (I). Chem Pharm Bull 1964, 12:1232-35.

27. Shibata S, Noguchi M: Two new coumarins in Boenninghausenia albiflora. Phytochemistry 1977, 16:291-93.

28. Rózsa Zs, Szendrei K, Kovacs Z, Novák I, Minker E, Reisch J: The cooccurrence of rutacridone and noracronycine in the roots of Boenninghausenia albiflora. Phytochemistry 1978, 17:169-70.

29. Rozsa Z, Szendrei K, Novak I, Reisch J, Minker E: Acridone alkaloids as constituents in the roots of Boenninghausenia albiflora. Pharmazie 1975, 30:753-4. 
30. Ohta T, Miyazaki T: Furoquinolines. XIV. Occurrence of dictamnine in Boenninghausenia albiflora avr. Japonica. Yakugaku Zasshi 1958, 78:1067-68.

31. Suga T, Shishibori T, Kosela S: The neutral volatiles of Boenninghausenia albiflora. Phytochemistry 1975, 14:308-309.

32. Fujita SI, Ishimatsu Y, Fugita Y: Miscellaneous contribution to the essential oils of the plants from various territories.XLII. On the components of the essential oils of Magnolia denudata Desr. Yakugaku Zasshi 1977, 97(II):1216-I8.

33. Miyazawa M, Tanaka S, Kameoka $\mathrm{H}$ : The constituent of the essential oil from Rumex japonicus Houtt. Yakugaku Zasshi 198I 101:660-62.

34. Shim SB, Kim NJ, Kim DH: Beta-glucuronidase inhibitory activity and hepatoprotective effect of 18 beta-glycyrrhetinic acid from the rhizomes of Glycyrrhiza uralensis. Planta Medica 2000 66(I):40-43

35. Jacobs BP, Dennehy C, Ramirez G, Sapp J, Lawrence VA: Milk thistle for the treatment of liver disease: A systematic review and meta-analysis. Am J Med 2002, I I 3:506-5 I5

36. Levy C, Seeff LD, Lindor KD: Use of herbal supplements for chronic liver disease. Clin Gastroenterol Hepatol 2004 2(I I):947-56

37. Kvasnicka F, Bba B, Sevck R, Voldrich M, Kratka J: Analysis of the active components of silymarin. J Chromatogr A 2003, 990(I2):239-245.

38. Bean P: The use of alternative medicine in the treatment of hepatitis C. Am Clin Lab 2002, 2 I (4):19-2I.

39. Hattori M: Metabolism of glycyrrhizin by human intestinal flora II. Isolation and characterization of human intestinal bacteria capable of metabolizing glycyrrhizin and related compounds. Chem Pharm Bull 1985, 33:210-17.

40. Akao T, Hattori M, et al.: Hydrolysis of glycyrrhizin to I 8 $\beta$-glycyrrhetyl monoglucuronide by lysosomal $\beta$-D-glucuronidase of animal livers. Biochem Pharmacol 1991, 4I: I025-9.

4I. Kim DH, Hong SW, Kim BT, Bae EA, Park HY, Han MJ: Biotransformation of glycyrrhizin by human intestinal bacteria and its relation to biological activities. Arch Pharm Res 2000 23(2): $172-7$

42. Sato H, Goto W, Yamamura J, Kurokawa M, Kageyama S, Takahara T, Watanabe A, Shiraki K: Therapeutic basis of glycyrrhizin on chronic hepatitis B. Antiviral Res 1996, 30(2-3): 171-7.

43. Wildhirt E: Experience in Germany with glycyrrhizinic acid for the treatment of chronic viral hepatitis. In Viral Hepatitis and Liver Disease Edited by: Nishioka K, Suzuki H, Mishiro S, Oda T. Tokyo: Springer-Verlag; 1994:658-61.

44. Arase Y, Ikeda K, Murashima N, Chayama K, Tsubota A, Koida I, Suzuki Y, Saitoh S, Kobayashi M, Kumada H: The long-term efficacy of glycyrrhizin in chronic hepatitis $\mathbf{C}$ patients. Cancer 1997, 79: |494-500.

45. Arase Y, Ikeda K, Murashima N, Chayama K, Tsubota A, Koida I, Suzuki Y, Saitoh S, Kobayashi M, Kumada H: Effect of a lignanenriched extract of Schisandra chinensis on aflatoxin BI and cadmium chloride-induced hepatotoxicity in rats. Pharmacol \& Toxicol 1996, 78(6):4I3-4I6.

46. Zhu M, Yeung RY, Lin KF, Li RC: Improvement of phase I drug metabolism with Schisandra chinensis against $\mathrm{CCl}_{4}$ hepatotoxicity in a rat model. Planta Medica 2000, 66(6):52I-25.

47. Ip SP, Ko KM: The crucial antioxidant action of schisandrin $B$ in protecting against carbon tetrachloride hepatotoxicity in mice: a comparative study with butylated hydroxytoluene. Biochem and Pharmacol 1996, 52:1687-93.

48. Wang JP, Raung SL, Hsu MF, Chen CC: Inhibition by gomisin C (a lignan from Schisandra chinensis) of the respiratory burst of rat neutrophils. $\mathrm{Br}$ J Pharmacol 1994, I I 3:945-53.

49. Yasukawa K, Ikeya Y, Mitsuhashi H, Iwasaki M, Aburada M, Nakagawa S, Takeuchi M, Takido M: Gomisin A inhibits tumor promotion by I 2-o-tetra-decanoylphorbol-13-acetate in two-stage carcinogenesis in mouse skin. Oncology 1992, 49:68-7I.

50. Fujihashi T, Hara H, Sakata T, Mori K, Higuchi H, Tanaka A, Kaji H, Kaji A: Anti-human immunodeficiency virus (HIV) activities of halogenated gomisin J derivatives, new nonnucleoside inhibitors of HIV type I reverse transcriptase. Antimicrob Agents Chemother 1995, 39(9):2000-7.

5I. IP SP, Ma CY, Che CT, Ko KM: Methylenedioxy group as a determinant of schisandrin in enhancing hepatic mitochondrial glutathione in carbon tetrachloride-intoxicated mice. Biochem Pharmacol 1997, 54:317-19.

52. Kim SR, Lee MK, Koo KA, Kim SH, Sung SH, Lee NG, Markelonis G], Oh TH, Yang JH, Kim YC: Dibenzocyclooctadiene lignans from Schisandra chinensis protect primary cultures of rat cortical cells from glutamate-induced toxicity. I Neurosci Res 2004, 76:397-405

53. Okamoto T, Kajino K, Hino O: Hepatoprotective drugs for the treatment of virus-induced chronic hepatitis: from hypercarcinogenic state to hypocarcinogenic state. Jpn J Pharmacol 200I, 87:I77-I80.

54. Okamoto T, Kobayashi T, Yoshida S: Chemical aspects of coumarin compounds for the prevention of hepatocellular carcinomas. Curr Med Chem Anticancer Agents 2005, 5(I):47-5I.

55. Ohtake N, Nakai Y, Yamamoto M, Sakakibara I, Takeda S, Amagaya S, Aburada $M$ : Separation and isolation methods for analysis of the active principles of Sho-saiko to (SST) oriental medicine. J Chromatogr B 2004, 8 | 2: I35-48.

56. Ono M, Miyamura M, Kyotani S, Saibara T, Ohnishi S, Nishioka Y: Effects of sho-saiko-to extract on liver fibrosis in relation to the changes in hydroxyproline and retinoid levels of the liver in rats. JPharm Pharmacol 1999, 5 I: I079- I084.

57. Wu H, Wang R, Kuo Cl, Lien LL, Lien EJ: SAR analysis of phytoandrogenic compounds in traditional medicines. Int J Orient Med 2003, 28(I): I3-34.

58. Lien El: Structure-activity correlations for anticonvulsant drugs. J Med Chem 1970, I3: I 189-91.

59. Lien EJ, Tong GL, Chou JT, Lien LL: Structural requirements for centrally acting drugs I. J Pharm Sci 1973, 62:246-250.

60. Lien EJ: The relationship between chemical structure and drug absorption, distribution and excretion. In Maas (ed) Medicinal Chemistry IV Proceedings of the 4th international symposium on medicinal chemistry Noordwijkerhout, The Netherlands: Elsevier Amsterdam; 1974:319-42.

61. Birnboim HC: Superoxide anion may trigger DNA strand breaks in human granulocytes by acting at a membrane target. In Ann N Y Acad Sci Volume 55I. Membrane in cancer cells :83-94. 30 December 1988 .

62. Hsu HY, Chen YP, Hong M, Eds: The Chemical Constituents of Oriental Herbal Drugs Volume I. Long Beach, CA: Oriental Healing Arts Institute of USA; 1982.

63. Hsu HY, Chen YP, Hong M, Eds: The Chemical Constituents of Oriental Herbal Drugs Volume 2. Long Beach, CA: Oriental Healing Arts Institute of USA; 1985.
Publish with Biomed Central and every scientist can read your work free of charge

"BioMed Central will be the most significant development for disseminating the results of biomedical research in our lifetime. "

Sir Paul Nurse, Cancer Research UK

Your research papers will be:

- available free of charge to the entire biomedical community

- peer reviewed and published immediately upon acceptance

- cited in PubMed and archived on PubMed Central

- yours - you keep the copyright
BiolMedcentral 